\title{
A COMPARISON PRINCIPLE FOR LARGE DEVIATIONS
}

\author{
JOHN R. BAXTER AND NARESH C. JAIN \\ (Communicated by William D. Sudderth)
}

\begin{abstract}
If $\left\{\mu_{n}\right\}$ and $\left\{\nu_{n}\right\}$ are two sequences of probability measures on a separable metric space, we give conditions under which $\left\{\mu_{n}\right\}$ satisfies a large deviation principle if and only if $\left\{\nu_{n}\right\}$ does. A known and a new theorem follow immediately from the application of this comparison principle to standard results in large deviation theory.
\end{abstract}

1. Introduction. Let $(S, d)$ be a metric space, where $d$ denotes the metric. $\mathscr{S}$ will denote the $\sigma$-algebra of Borel subsets of $S$. A sequence of probability measures on $(S, \mathscr{S})$ is said to satisfy a large deviation principle (LDP) with rate function $I$, if

(1.1) for every closed set $F$

$$
\limsup _{n \rightarrow \infty}(1 / n) \log \mu_{n}(F) \leq-\inf _{x \in F} I(x)
$$

and

(1.2) for every open set $G$

$$
\liminf _{n \rightarrow \infty}(1 / n) \log \mu_{n}(G) \geq-\inf _{x \in G} I(x) .
$$

The rate function $I$ is a function from $S$ to $[0, \infty]$ which satisfies

(1.3a) $I(x)$ is not identically $\infty, I$ is lower semicontinuous,

(1.3b) $\{I \leq a\}$ is compact for all $a<\infty$.

We will observe in $\S 2$ (Propositions 1 and 2) that if $\left\{\mu_{n}\right\}$ and $\left\{\nu_{n}\right\}$ are two sequences of probability measures on $S$ which become sufficiently close as $n \rightarrow \infty$, then $\left\{\mu_{n}\right\}$ satisfies the LDP with rate function $I$ if and only if $\left\{\nu_{n}\right\}$ does. We then apply these simple observations to obtain a comparison principle for the large deviations of the sample mean when the summands are independent and identically distributed (i.i.d.) and take values in a locally convex topological vector space (Theorem 1). Propositions 1, 2, and Theorem 1 are the main results of this note.

The motivation for Theorem 1 becomes clear in $\S 3$, where two applications are given. We first state two standard results on large deviations as Theorems 2 and 3 in $\S 3$ for ready reference. These theorems are extensions of the classical Cramér and Sanov theorems by Donsker and Varadhan [3]. Theorems 1 and 2 then yield Theorem 4 (due to Bolthausen [1]) as an immediate corollary. Likewise, Theorems 1 and 3 immediately give the corresponding perturbation of Theorem 3 without any additional work. Bolthausen's theorem [1] is a generalization of a theorem

Received by the editors July 16, 1987.

1980 Mathematics Subject Classification (1985 Revision). Primary 60F10, 60G15, 60B12.

Key words and phrases. Large deviations, Banach-space-valued Random Variable.

This work was partly supported by NSF Grant DMS86-03437. 
of Chevet [2]. For applications of such perturbations of standard large deviation results one may consult Ellis and Rosen [5].

For future use, we state the definition of the Prohorov distance between two probability measures $\mu, \nu$ on $(S, d)$.

$$
\rho(\mu, \nu)=\inf \left\{\delta>0: \mu(F) \leq \nu\left(F^{\delta}\right)+\delta \text { for all closed sets } F\right\},
$$

where for any set $A \subset S, \delta>0$,

$$
A^{\delta}=\{y: d(x, y)<\delta \text { for some } x \in A\}
$$

If different metrics are used, we will write $A^{\delta}(d)$ to indicate that the metric $d$ is under consideration.

For $\varepsilon>0$, we also define

$$
\rho_{\varepsilon}(\mu, \nu)=\inf \left\{\delta>0: \mu(F) \leq \nu\left(F^{\varepsilon}\right)+\delta \text { for all closed sets } F\right\} .
$$

It is easy to check that $\rho^{\varepsilon}(\mu, \nu)=\rho^{\varepsilon}(\nu, \mu)$.

Let $C_{b}(S)$ denote the continuous bounded functions on $S$. As usual, we say that a sequence of probabilities $\mu_{n}$ on $S$ converges weakly to a limit $\mu$ if $\int_{S} f d \mu_{n} \rightarrow$ $\int_{S} f d \mu$, for every $f \in C_{b}(S)$. We will denote the set of all probability measures on $S$ by $\mathscr{M}_{1}(S)$, and consider $\mathscr{M}_{1}(S)$ to be a topological space with the topology corresponding to weak convergence. For a sequence of probability measures, convergence in the Prohorov distance implies weak convergence, and when the underlying space $S$ is separable, the converse holds. Thus for separable $S, \mathscr{M}_{1}(S)$ is a separable metric space with the Prohorov distance as its metric.

In what follows we will use $\delta_{x}$ to denote the Dirac measure with unit mass at $x$.

\section{The main results.}

PROPOSITION 1. Let $\left\{\mu_{n}\right\},\left\{\nu_{n}\right\}$ be two sequences of probability measures on a metric space $(S, d)$ such that for every closed set $F$ and every $\varepsilon>0$

$$
\begin{aligned}
& \limsup _{n \rightarrow \infty}(1 / n) \log \mu_{n}(F) \leq \limsup _{n \rightarrow \infty}(1 / n) \log \nu_{n}\left(F^{\varepsilon}\right), \\
& \liminf _{n \rightarrow \infty}(1 / n) \log \nu_{n}(F) \leq \liminf _{n \rightarrow \infty}(1 / n) \log \mu_{n}\left(F^{\varepsilon}\right) .
\end{aligned}
$$

Then the LDP holds for $\left\{\mu_{n}\right\}$ with rate function I if it holds for $\left\{\nu_{n}\right\}$ with the same rate function.

For the proof of Proposition 1 we need Lemma 1 below. As a convenient notation, for any set $A$ we will define

$$
I(A)=\inf _{x \in A} I(x) .
$$

LEMMA 1. If $F$ is closed, and $I$ is a rate function (satisfying (1.3)), then

$$
I(F)=\lim _{\delta \rightarrow 0} I\left(F^{\delta}\right) \text {. }
$$

This lemma is well known, and the proof is easy. Note that $(1.3 \mathrm{~b})$ is used in the proof of this lemma.

PROOF OF PROPOSITION 1. Assume the LDP holds for $\left\{\nu_{n}\right\}$ with rate function $I$. Let $F$ be a closed set. Since the LDP holds for $\left\{\nu_{n}\right\}$ with rate $I$, we have by (2.1a) that

$$
\limsup _{n \rightarrow \infty}(1 / n) \log \mu_{n}(F) \leq I\left(F^{\varepsilon}\right)
$$


for all $\varepsilon>0$, and by Lemma 1 we get (1.1) by letting $\varepsilon \rightarrow 0$.

If $G$ is an open set, to prove (1.2) we may assume without any loss of generality that $I(G)<\infty$. Let $I(G)=\beta$. Fix $\alpha>\beta$. Then there is a point $x \in G$ with $I(x)<\alpha$. Let $B$ be an open ball around $x, \varepsilon>0$ such that $D^{\varepsilon} \subset G$, where $D$ is the closure of $B$. We have $\mu_{n}(G) \geq \mu_{n}\left(D^{\varepsilon}\right)$. Also, by $(2.1 \mathrm{~b})$

$$
\liminf _{n \rightarrow \infty}(1 / n) \log \nu_{n}(D) \leq \liminf _{n \rightarrow \infty}(1 / n) \log \mu_{n}\left(D^{\varepsilon}\right) .
$$

Therefore

$$
\liminf _{n \rightarrow \infty}(1 / n) \log \mu_{n}(G) \geq \liminf _{n \rightarrow \infty}(1 / n) \log \nu_{n}(B) \geq-I(B) \geq-\alpha .
$$

Thus (1.2) holds and Proposition 1 is proved.

PROPOSITION 2. Let $\left\{\mu_{n}\right\},\left\{\nu_{n}\right\}$ be two sequences of probability measures on a metric space $(S, d)$ such that for every $\varepsilon>0$

$$
\lim _{n \rightarrow \infty}(1 / n) \log \rho_{\varepsilon}\left(\mu_{n}, \nu_{n}\right)=-\infty
$$

Then the LDP holds for $\left\{\mu_{n}\right\}$ with rate function $I$ if and only if it holds for $\left\{\nu_{n}\right\}$ with the same rate function.

ProOF. For any $a, b>0$,

$$
\log (a+b) \leq \log 2+\max (\log a, \log b) .
$$

For any closed set $F$, and any $\varepsilon>0$, by definition (recall that $\rho_{\varepsilon}$ is symmetric) $\mu_{n}(F) \leq \nu_{n}\left(F^{\varepsilon}\right)+\rho_{\varepsilon}\left(\mu_{n}, \nu_{n}\right)$ and $\nu_{n}(F) \leq \mu_{n}\left(F^{\varepsilon}\right)+\rho_{\varepsilon}\left(\mu_{n}, \nu_{n}\right)$. (2.1a) and (2.1b) follow at once, and since we may exchange $\mu_{n}$ and $\nu_{n}$, Proposition 2 is proved.

Before stating Theorem 1, we recall the notion of a locally convex topological vector space. $E$ is a locally convex vector space if there is a family of seminorms $\left\{\|\cdot\|_{j}\right\}_{j \in J}$ separating points on $E$, which defines the topology on $E$. We will assume that $E$ is metrizable, or equivalently that the index set $J$ is countable, say $J=\{1,2, \ldots\}$. We also assume that $E$ is separable. Let $\mathscr{E}$ denote the Borel sets of $E$. We will denote by $d_{j}$ the metric on $E$ defined by

$$
d_{j}(x, y)=\|x-y\|_{j} /\left(1+\|x-y\|_{j}\right)
$$

and let

$$
\bar{d}=\sum_{j=1}^{\infty} 2^{-j} d_{j}
$$

The metric $\bar{d}$ is a convenient metric for the topology on $E$.

If $\left\{\mu_{n}\right\}$ is a sequence of probability measures on $(E, \mathscr{E})$, for each $n$ we will denote by $\bar{\mu}_{n}$ the probability measure on $(E, \mathscr{E})$ defined by

$$
\bar{\mu}_{n}(A)=\mu_{n}^{* n}(n A), \quad A \in \mathscr{E},
$$

where $\mu^{* k}$ denotes the $k$-fold convolution of $\mu$. Note that if $X_{n 1}, X_{n 2}, \ldots, X_{n n}$ are i.i.d. with distribution $\mu_{n}$, then $\bar{\mu}_{n}$ denotes the distribution of $\left(X_{n 1}+\cdots+X_{n n}\right) / n$. 
THEOREM 1. Let $E$ be a locally convex topological vector space as above. Let $\left\{\mu_{n}\right\},\left\{\nu_{n}\right\}$ be two sequences of probability measures on $(E, \mathscr{E})$ such that $\rho\left(\mu_{n}, \nu_{n}\right) \rightarrow$ 0 as $n \rightarrow \infty$, and such that for every $j$ and for each $t \geq 0$, the sequences $\int e^{t\|x\|_{j}} d \mu_{n}$ and $\int e^{t|| x \|_{j}} d \nu_{n}$ are bounded in $n$. Then $\left\{\bar{\mu}_{n}\right\}$ obeys the large deviation principle with rate function $I$ if and only if $\left\{\bar{\nu}_{n}\right\}$ does.

PROOF. It is enough to show that (2.4) holds, with $S=E, d=\bar{d}, \mu_{n}=\bar{\mu}_{n}$ and $\nu_{n}=\bar{\nu}_{n}$. Let $F$ be a closed set, $\varepsilon>0$. Let $j$ be sufficiently large that if $d^{\prime}=d_{1}+\cdots+d_{j}$ then $\bar{d}<d^{\prime}+\varepsilon / 2$. Then $F^{\varepsilon}(\bar{d}) \supset F^{\varepsilon / 2}\left(d^{\prime}\right)$. Thus it is enough to prove (2.4) when $d=d^{\prime}$. Let $\|\cdot\|=\|\cdot\|_{1}+\cdots+\|\cdot\|_{j}$, and let $d^{\prime \prime}(x, y)=\|x-y\|$. Clearly $d^{\prime} \leq d^{\prime \prime}$. Thus $F^{\varepsilon}\left(d^{\prime \prime}\right) \subset F^{\varepsilon}\left(d^{\prime}\right)$. Thus it is enough to prove (2.4) when $d=d^{\prime \prime}$.

We now define a coupling of $\mu_{n}, \nu_{n}$. Since $\rho\left(\mu_{n}, \nu_{n}\right) \rightarrow 0$, by an extension of a theorem of Strassen, due to Dudley (cf. [4, Corollary 18.3]) we know that for each $n$ we can find $E$-valued random variables $X_{n}, Y_{n}$ (on some probability space), such that $X_{n}$ has distribution $\mu_{n}, Y_{n}$ has distribution $\nu_{n}$, and $\bar{d}\left(X_{n}, Y_{n}\right) \rightarrow 0$ in probability as $n \rightarrow \infty$. Hence $\left\|X_{n}-Y_{n}\right\| \rightarrow 0$ in probability as $n \rightarrow \infty$. For each $n$, let $\left(X_{n}(j), Y_{n}(j)\right)$ be a sequence of independent $E \times E$-valued random variables such that for every $j,\left(X_{n}(j), Y_{n}(j)\right)$ has the same distribution as $\left(X_{n}, Y_{n}\right)$. Let $S_{n}=X_{n}(1)+\cdots+X_{n}(n), T_{n}=Y_{n}(1)+\cdots+Y_{n}(n), Z_{n}(j)=\left\|X_{n}(j)-Y_{n}(j)\right\|$, and $V_{n}=Z_{n}(1)+\cdots+Z_{n}(n)$. Let $c_{n}(t)$ denote $E\left(e^{t Z_{n}(j)}\right)$. Then $c_{n}(t) \leq \int e^{2 t|| x \|} d \mu_{n}+$ $\int e^{2 t \mid\|x\|} d \nu_{n}$. It follows easily that $\left\{e^{t Z_{n}(j)}: n \geq 1\right\}$ is uniformly integrable. Since $Z_{n}(j) \rightarrow 0$ in probability as $n \rightarrow \infty$, we have $c_{n}(t) \rightarrow 1$ as $n \rightarrow \infty$, for all $t \geq 0$.

For any set $A \in \mathscr{E}$, for any $\varepsilon>0$,

$$
\bar{\mu}_{n}(A)=P\left(S_{n} \in n A\right) \leq P\left(T_{n} \in n A^{\varepsilon}\right)+P\left(V_{n} \geq n \varepsilon\right) .
$$

Since $E e^{t V_{n}}=\left(c_{n}(t)\right)^{n}, P\left(V_{n} \geq n \varepsilon\right) \leq e^{-n \varepsilon t}\left(c_{n}(t)\right)^{n}$, we have

$$
\bar{\mu}_{n}(A) \leq \bar{\nu}_{n}\left(A^{\varepsilon}\right)+e^{-n \varepsilon t}\left(c_{n}(t)\right)^{n} .
$$

Thus $(1 / n) \log \rho_{\varepsilon}\left(\bar{\mu}_{n}, \bar{\nu}_{n}\right) \leq-\varepsilon t+\log c_{n}(t)$. Hence, since $t$ is arbitrary, we see easily that (2.4) holds, so Theorem 1 is proved.

REMARKS. If the sequence $\left\{\nu_{n}\right\}$ in Theorem 1 is constant, i.e. $\nu_{n}=\nu$ for every $n$, then the condition $\rho\left(\mu_{n}, \nu_{n}\right) \rightarrow 0$ simply says that $\mu_{n} \rightarrow \nu$ weakly. Let $C_{b}^{j}(E)$ denote the space of bounded functions on $E$ which are continuous with respect to the norm $\|\cdot\|_{1}+\cdots+\|\cdot\|_{j}$. It is easy to see that the union of the spaces $C_{b}^{j}(E)$ is strongly separating and hence forms a convergence determining class. Thus when $\nu_{n}=\nu$ for all $n$, to check that $\rho\left(\mu_{n}, \nu_{n}\right) \rightarrow 0$ it is enough to check that for every $j$

$$
\int_{E} f d \mu_{n} \rightarrow \int_{E} f d \nu, \quad f \in C_{b}^{j}(E) .
$$

3. Applications. We first state two standard results from [3].

THEOREM 2. Let $E$ be a separable Banach space with dual $E^{*}$, and let $\mu$ be a probability measure on $E$. If $\int \exp (t|| x \|) d \mu(x)<\infty$ for every $t>0$, then $\bar{\mu}_{n}$ (defined by (2.5) with $\mu_{n} \equiv \mu$ ) satisfies the LDP with rate function

$$
I(x)=\sup _{\theta \in E^{*}}\left\{\langle\theta, x\rangle-\log \int \exp (\langle\theta, y\rangle) d \mu(y)\right\} .
$$


THEOREM 3. Let $(S, d)$ be a complete separable metric space, and let $X_{1}, X_{2}, \ldots$ be independent random variables with values in $S$ and having common distribution $\alpha$. Let $\bar{\mu}_{n}$ denote the distribution of $\left(\delta_{X_{1}}+\cdots+\delta_{X_{n}}\right) / n$ in $\mathscr{M}_{1}(S)$. Then $\left\{\bar{\mu}_{n}\right\}$ satisfies the LDP with rate function $I_{\alpha}(\beta)$, where

$$
I_{\alpha}(\beta)=\int \log (d \beta / d \alpha) d \beta
$$

if $\beta \ll \alpha$ and $\int|\log (d \beta / d \alpha)| d \beta<\infty ; I_{\alpha}(\beta)=\infty$, otherwise.

The next theorem is due to Bolthausen [1] and we will show how it follows directly from Theorems 1 and 2 .

THEOREM 4. Let $E$ be a separable Banach space. Let $\left\{\mu_{n}\right\}$ be a sequence of probability measures on $E$ such that $\mu_{n} \rightarrow \mu$ weakly. If for every $t>0$, $\int \exp (t|| x \|) d \mu(x)$ is bounded in $n$, then $\bar{\mu}_{n}$ defined in (2.5) satisfies the LDP with rate function I defined by (3.1).

ProOF. In Theorem 1 we take $\|\cdot\|_{j}=\|\cdot\|$, the Banach norm, for every $j$. Let $\nu_{n}=\mu$. Theorem 1 then says that $\left\{\bar{\mu}_{n}\right\}$ satisfies the LDP if $\hat{\mu}_{n}$ does, and with the same rate function, where

$$
\bar{\mu}_{n}(A)=\mu_{n}^{* n}(n A), \quad \text { and } \quad \hat{\mu}_{n}(A)=\mu^{* n}(n A) .
$$

The rest then follows from Theorem 2 , and the proof is complete.

The next theorem is a perturbation of Theorem 3, in the same sense that Theorem 4 is a perturbation of Theorem 2 .

THEOREM 5. Let $(S, d)$ be a complete separable metric space. Let $\left\{\alpha_{n}\right\}$ be a sequence of probability measures on $(S, d)$ such that $\alpha_{n} \rightarrow \alpha$ weakly. For each $n$, let $X_{1}(n), X_{2}(n), \ldots, X_{n}(n)$ be $S$-valued independent random variables with common distribution $\alpha_{n}$. Let $\bar{\mu}_{n}$ denote the distribution of $\left(\delta_{X_{1}(n)}+\cdots+\delta_{X_{n}(n)}\right) / n$ on $\mathscr{M}_{1}(S)$. Then $\left\{\bar{\mu}_{n}\right\}$ satisfies the LDP with rate function $I_{\alpha}$ given by (3.2).

PROOF. Let $\left\{f_{j}\right\}_{j \geq 1}$ be a convergence-determining class in $\mathscr{C}_{b}(S)$. We may choose each $f_{j}$ to be uniformly continuous with $0 \leq f_{j} \leq 1$. The topology of weak convergence on $\mathscr{M}_{1}(S)$ is given by the metric

$$
\hat{\rho}\left(\nu_{1}, \nu_{2}\right)=\sum_{j=1}^{\infty} 2^{-j}\left|\int f_{j} d \nu_{1}-\int f_{j} d \nu_{2}\right| .
$$

Let $E$ denote the linear space of linear functionals on the span of the $f_{j}$. Define seminorms $\|\cdot\|_{j}$ on $E$ by $\|\varphi\|_{j}=\left|\varphi\left(f_{j}\right)\right|, j \geq 1$. Then $E$ satisfies the conditions of Theorem 1 . We may regard $\mathscr{M}_{1}(S)$ as a subset of $E$, since a measure $\nu$ gives a linear functional $\varphi_{\nu}(f)=\int f d \nu$, and a measure $\nu$ in $\mathscr{M}_{1}(S)$ is uniquely determined by the values $\varphi_{\nu}\left(f_{j}\right), j=1,2, \ldots$. It is easy to see that the topology induced on $\mathscr{M}_{1}(S)$ by $E$ is the weak topology.

Let $\psi: S \rightarrow \mathscr{M}_{1}(S)$ be the map $\psi(x)=\delta_{x} . \psi$ is continuous. Let $\mu_{n} \equiv \alpha_{n} \circ \psi^{-1}$, $\mu \equiv \alpha \circ \psi^{-1}$. Then $\mu_{n} \rightarrow \mu$ weakly as $n \rightarrow \infty$. We can extend the measures $\bar{\mu}_{n}$, $\mu_{n}, \mu$ from $\mathscr{M}_{1}(S)$ to $E$ in the usual way, by setting each measure equal to 0 on $E-\mathscr{M}_{1}(S)$. Clearly we still have $\mu_{n} \rightarrow \mu$ weakly as $n \rightarrow \infty$. Hence $\rho^{\prime}\left(\mu_{n}, \mu\right) \rightarrow 0$, where $\rho^{\prime}$ is the Prohorov distance with respect to the metric $\bar{d}=\sum_{j=\infty}^{\infty} 2^{-j} d_{j}$ defined in $\S 2$. Since $\delta_{X_{j}(n)}$ has distribution $\mu_{n}, \bar{\mu}_{n}$ and $\mu_{n}$ satisfy (2.5). We extend 
$I_{\alpha}$ to $E$ by setting $I_{\alpha}(\varphi)=\infty$ if $\varphi$ is not in $\mathscr{M}_{1}(S)$. Let $\bar{\nu}_{n}(A)=\mu^{* n}(n A), A \in \mathscr{E}$. Then the sequence $\left\{\bar{\nu}_{n}\right\}$ satisfies the LDP on $\mathscr{M}_{1}(S)$ with rate function $I_{\alpha}$, by Theorem 3. It is trivial to see that then $\left\{\bar{\nu}_{n}\right\}$ satisfies the LDP on $E$ with rate function $I_{\alpha}$. We note that $\int \exp \left(t|| x \|_{j}\right) d \mu_{n}(x)=\int \exp \left(t f_{j}\right) d \alpha_{n} \leq e^{t}$. Thus the conditions of Theorem 1 hold, and hence $\left\{\bar{\mu}_{n}\right\}$ satisfies the LDP with rate function $I_{\alpha}$ on $E$, and in particular on $\mathscr{M}_{1}(S)$, so Theorem 5 is proved.

REMARK. We assumed that $(S, d)$ was complete separable in Theorem 5 , because Theorem 3 requires this assumption. If we wished to state Theorem 5 as a comparison principle for two general sequences $\alpha_{n}$ and $\beta_{n}$, the completeness condition could be dropped, since only Theorem 1 is needed in this case. The proof of this comparison principle is very little different from the argument given above, except that one needs to note that the map $\psi$ is uniformly continuous between the two metrics used, and thus if $\rho\left(\alpha_{n}, \beta_{n}\right) \rightarrow 0$, where $\rho$ denotes the Prohorov distance using $d$, then $\rho^{\prime}\left(\alpha_{n} \psi^{-1}, \beta_{n} \psi^{-1}\right) \rightarrow 0$, where $\rho^{\prime}$ denotes the Prohorov distance using $\bar{d}$.

ACKNOWLEDGEMENT. We are thankful to S. R. S. Varadhan for the formulation of condition (2.4) in Proposition 2.

\section{REFERENCES}

1. E. Bolthausen, On the probability of large deviations in Banach spaces, Ann. Probab. 12 (1984), 427-435.

2. S. Chevet, Gaussian measures and large deviations, Probability in Banach Spaces. IV, Oberwolfach 1982, Lecture Notes in Math., vol. 990, Springer-Verlag, Berlin and New York, 1983, pp. 30-46.

3. M. D. Donsker and S. R. S. Varadhan, Asymptotic evaluation of certain Markov process expectations for large time. III, Comm. Pure Appl. Math. 29 (1976), 389-461.

4. R. M. Dudley, Convergence of laws on metric spaces, with a view to statistical testing, Lecture Notes No. 45, Matematisk Institut, Aarhus Universitet, Denmark, 1976.

5. R. S. Ellis and J. S. Rosen, Laplace's method for Gaussian integrals with an application to statistical mechanics, Ann. Probab. 10 (1982), 47-66.

6. S. R. S. Varadhan, Large deviations and applications, CBMS-NSF Regional Conf. Ser. in Appl. Math., SIAM, Philadelphia, Pa., 1982.

School of Mathematics, University of Minnesota, Minneapolis, Minnesota 55455 